\title{
Detection of BCG bacteria using a magnetoresistive biosensor: A step towards a fully electronic platform for tuberculosis point-of-care detection
}

\author{
Teresa G. Barroso ${ }^{\mathrm{a}, \mathrm{b}, \mathrm{c}, *}$, Rui C. Martins ${ }^{\mathrm{d}}$, Elisabete Fernandes ${ }^{\mathrm{c}}$, Susana Cardoso ${ }^{\mathrm{e}}$, José Rivas ${ }^{\mathrm{c}, \mathrm{f}}$, \\ Paulo P. Freitas ${ }^{\mathrm{c}, \mathrm{e}}$ \\ a ICVS: Life and Health Sciences Research Institute, School of Health Sciences, University of Minho, Campus de Gualtar, 4710-057 Braga, Portugal \\ b ICVS/3B's - PT: Government Associate Laboratory, Braga/ Guimarães, Campus de Gualtar, 4710-057 Braga, Portugal \\ c INL: International Iberian Nanotechnology Laboratory, Avenida Mestre José Veiga s/n, 4715-330 Braga, Portugal \\ d INESC TEC, Campus da FEUP, Rua Dr. Roberto Frias, 4200-465 Porto, Portugal \\ e INESC MN, Rua Alves Redol 9, 1000 Lisbon, Portugal \\ f Department of Applied Physics, Universidade de Santiago de Compostela, Rúa Xosé María Suárez Núñez, s/n. Campus Vida, E- 15782, Santiago de \\ Compostela, Spain
}

\section{A R T I C L E I N F O}

\section{Keywords:}

Tuberculosis

Nanotechnology

Magnetic nanoparticles

Magnetoresistive biosensor

Chemometrics

\begin{abstract}
A B S T R A C T
Tuberculosis is one of the major public health concerns. This highly contagious disease affects more than 10.4 million people, being a leading cause of morbidity by infection. Tuberculosis is diagnosed at the point-of-care by the Ziehl-Neelsen sputum smear microscopy test. Ziehl-Neelsen is laborious, prone to human error and infection risk, with a limit of detection of $10^{4}$ cells $/ \mathrm{mL}$. In resource-poor nations, a more practical test, with lower detection limit, is paramount. This work uses a magnetoresistive biosensor to detect BCG bacteria for tuberculosis diagnosis. Herein we report: i) nanoparticle assembly method and specificity for tuberculosis detection; ii) demonstration of proportionality between BCG cell concentration and magnetoresistive voltage signal; iii) application of multiplicative signal correction for systematic effects removal; iv) investigation of calibration effectiveness using chemometrics methods; and v) comparison with state-of-the-art point-of-care tuberculosis biosensors.

Results present a clear correspondence between voltage signal and cell concentration. Multiplicative signal correction removes baseline shifts within and between biochip sensors, allowing accurate and precise voltage signal between different biochips. The corrected signal was used for multivariate regression models, which significantly decreased the calibration standard error from 0.50 to $0.03 \log _{10}$ (cells $/ \mathrm{mL}$ ). Results show that Ziehl-Neelsen detection limits and below are achievable with the magnetoresistive biochip, when pre-processing and chemometrics are used.
\end{abstract}

\section{Introduction}

Tuberculosis (TB) is a major global health concern and a destructive disease, associated with high morbidity in 2015: 10.4 million people, including 11\% HIV-positive patients. Moreover, TB was reported as a leading cause of mortality from an infectious agent, causing the death of 1.4 million people (among 0.4 million HIV-positive) (WHO, 2016).

The impact of Mycobacterium tuberculosis (Mtb) bacterial infection is particularly significant in resource-limited countries, which represent more than $80 \%$ of global epidemics (WHO, 2015, 2016). In these settings, direct Ziehl-Neelsen (ZN) sputum smear microscopy is an important diagnostic technique, although the method is laborious and prone to human error and to infection exposure (Yeo et al., 2009;
Matu and Juma, 2013; Creecy et al., 2015). ZN only identifies TB pulmonary cases in sputum samples, with a limit of detection (LOD) of $10^{4}$ cells/mL (Arias-Bouda et al., 2000; Yeo et al., 2009; Creecy et al., 2015). Therefore, active TB cases with lower number of cells, patients with negative sputum results, children, HIV patients and extrapulmonary TB, need a different diagnostic approach (Moreno-Pérez et al., 2010; Parsons et al., 2011; Veigas et al., 2012; WHO, 2012). Inexpensive point-of-care (POC) tests that can be used with blood or urine, are extremely necessary (Arias-Bouda et al., 2000; Yeo et al., 2009; Lange and Mori, 2010).

Immunoassays provide simple, inexpensive and rapid diagnosis, including for people with negative sputum samples and immunosuppressed patients (Perkins and Cunningham, 2007; Lange and Mori,

\footnotetext{
* Corresponding author at: INL: International Iberian Nanotechnology Laboratory, Avenida Mestre José Veiga s/n, 4715-330 Braga, Portugal

E-mail address: teresa.barroso@gmail.com (T.G. Barroso).
} 
a

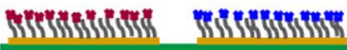

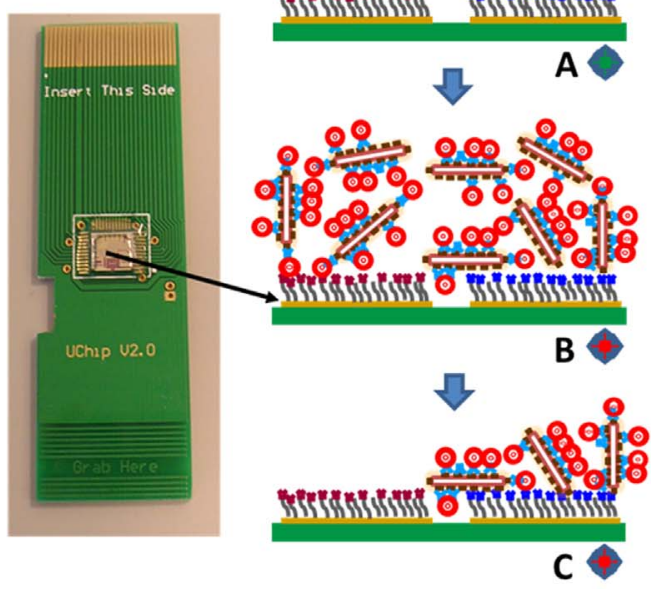

b

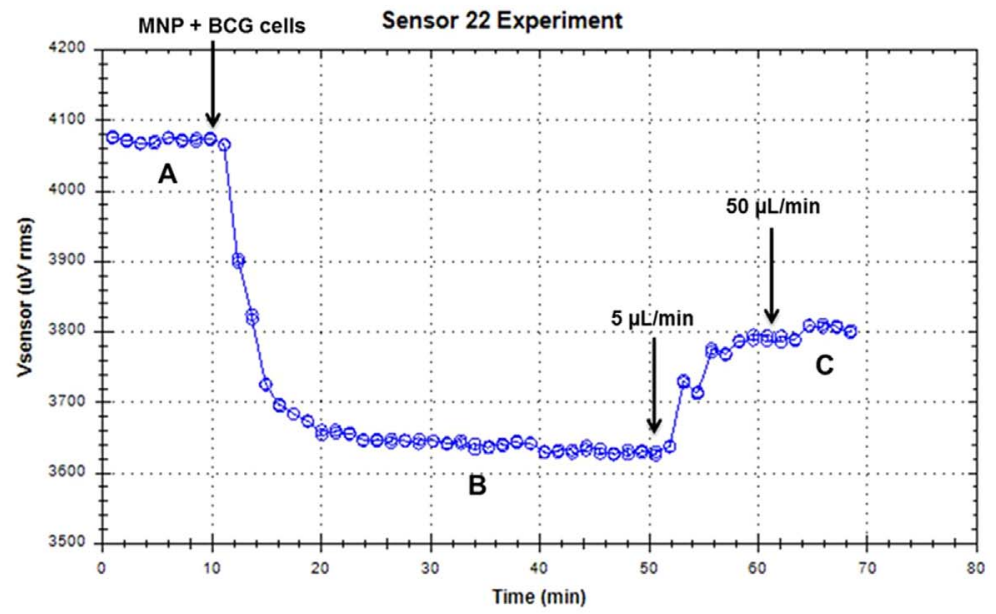

BCG bacteria; - BCG antigen; - MNP; Ydetection Abs; Ycontrol Abs; Ycapture Abs;

\SPDP linker; MR sensor; MR biochip; - MR signal; + MR signal.

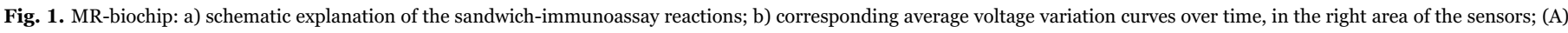

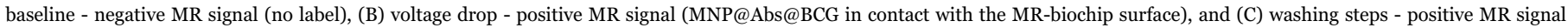
(MNP@Abs@BCG binded to specific Abs on the biochip surface).

2010). Magnetoresistive (MR) biosensors can replace the enzymatic detection label of immunosorbent assays (ELISA) with magnetic nanoparticles (MNP), functionalized with specific antibodies (Abs) and bioconjugated with bacterial cells (Fernandes et al., 2014; Barroso et al., 2015). On the biochip surface, capture Abs bind specific antigens on bacterial samples. Magnetically labelled cells are detected by an array of spin-valve sensors, which are sensitive to small changes in the magnetic field of MNP (Germano et al., 2009; Martins et al., 2009; Freitas et al., 2012; Giouroudi and Keplinger, 2013; Barroso et al., 2015).

Fig. 1a presents the time course voltage signal variation of the MRbiosensor, into three steps: A) Similarly to a common ELISA, the surface is functionalized with specific capture Abs (right area of the sensor) that bind matching antigens; control Abs are chemically attached to the surface (left area) validating the specificity of the platform; when no label is on the surface of the biosensor, a baseline MR signal is registered $\left(\mathrm{V}_{\text {sensor }}\right)$; $\left.\mathrm{B}\right)$ Then, the immunoassay follows a sandwich format labelled by MNP functionalized with detection Abs (anti-Mtb bio) and bioconjugated with target bacterial cells; when MNP get near the biochip surface, a MR change is created by the magnetic stray fields; this signal is detected by the sensors and a voltage drop is observed; C) After $30 \mathrm{~min}$ of contact with the biochip surface, nonbound MNP are washed out, and only the signal from labelled targets is recorded $\left(\mathrm{V}_{\text {particles }}\right)$.

For TB detection in blood, sputum or urine samples, which can contain Mtb bacteria, it is necessary to remove all other components to obtain a clean sample that can be injected into the MR-biochip. This can be done by magnetic separation. Once magnetically-labelled Abs are added to the sample, they specifically bind with mycobacteria, which can be magnetically separated. Then, this purified solution is injected into the biochip microfluidics. Magnetically functionalized bacterial cells are captured by specific Abs on the MR-biochip surface. The MR signal changes with time, as more cells attach to the surface (step B - Fig. 1b). The rate of voltage change is proportional to cell concentration. Once all MNP are bound to the biochip surface, there is no longer a voltage variation. At this point, washing cycles are necessary to remove non-bound MNP, only obtaining a voltage of the bound mycobacteria. The mycobacterial cell concentration is afterwards related to the differential between voltage at baseline (step A Fig. 1b) and the washing step (step C - Fig. 1b) (Martins et. al, 2009;
Barroso et al., 2015).

MR-biosensors have shown promising characteristics, such as, high sensitivity, fast performance, flexibility, electronic platform compatibility, integrability, miniaturization, scalability and portability (Martins et al., 2005, 2009, 2010; Germano et al., 2009; Freitas et al., 2012, 2016; Fernandes et al., 2014). MR-biosensors were able to detect low concentrations of DNA samples and bacterial cells (Martins et al., 2009; Fernandes et al., 2014). A femtomolar LOD was obtained by testing 20 mer ssDNA hybridization events (Martins et al., 2009) and 3-4 cells of Salmonella Enteritidis per sensor were detected, by immobilizing bacteriophages on the surface of the MRbiochip (Fernandes et al., 2014).

Nevertheless, MR-biosensors are prone to systematic artifacts. These include surface-imposed heterogeneity at the sensor spots (Abs probe inhomogeneity), diffusional effects, avidity, saturation, matrix effects and non-specific binding (Morton and Myszka, 1998; Myszka, 1999; Zhang et al., 2006; Martins et al., 2009, 2012; Barroso et al., 2015). These can be minimized by improving: i) biochip platform processing hardware (Germano et al., 2009); ii) experimental methods (Martins et al., 2009; Barroso et al., 2015); and iii) data processing (Myszka, 1999).

Myszka provides detailed information on minimizing systematic errors in biochip measurements (Myszka, 1999). For MR-biosensors, the following can be highlighted:

1. Biochip design and operation variables: i) homogeneity of immobilized Abs; and ii) sample flow and washing. Non-homogeneous distribution of immobilized Abs decreases reproducibility between different biochips, because the number of binding sites is not constant. Binding sites saturation sets the maximum concentration that the biochip can measure, which can be managed by changing the sample flow and time for binding reaction. However, in practice, constant flow rates and reaction times are established for allowing measurement of a range of cell concentrations and the removal of unbound material on the biochip surface, ensuring efficient and repeatable procedures (Martins et al., 2012; Barroso et al., 2015).

2. Experimental design: i) reagents and nanoparticles quality; ii) specificity; iii) concentration and washing; iv) avidity; and v) oriented immobilization. For example, washing buffers characteristics ( $\mathrm{pH}$, concentration, salinity), type of Abs (specificity), concen- 
tration of Abs and bacteria, and type of surface immobilization linker, are crucial to the success of MR detection (Martins et al., 2009, 2010; Barroso et al., 2015).

3. Data processing for artifacts removal: i) baseline correction; ii) multiplicative effects; iii) replication for determining bias and precision, as well as, removing white noise. Biochips present poor baseline reproducibility due to manufacturing limitations. Many authors recommend 'zeroing', that is, standardizing the voltage signal in each sensor by subtracting the first baseline values to the biochip signal (Myszka, 1999). This is not enough when the signal has multiplicative effects due to biochip design and distribution of immobilized antibodies. Other authors apply normalization for comparing signals with different baselines (Martins et al., 2009; Fernandes et al., 2014; Barroso et al., 2015), which is also not very effective in removing systematic artifacts.

The main goal of this study is to provide a proof-of-concept MRbiosensor for TB POC diagnosis. This research aims to: i) develop a robust pre-processing technique for effectively removing systematic effects on the voltage signal; ii) study the effectiveness of chemometrics, for providing an automatic quantification method; and iii) investigate how LOD is affected by these different approaches.

\section{Materials and methods}

\subsection{Reagents}

Ultrapure water and analytical grade reagents were used in all the experiments. The reagents for buffer preparation were purchased from Sigma: phosphate buffer (PB; $100 \mathrm{mM} \mathrm{NaH} \mathrm{PO}_{4}, 100 \mathrm{mM} \mathrm{Na}_{2} \mathrm{HPO}_{4}$, pH 7.4), TE buffer (10 mM Tris-HCl, 1 mM EDTA, pH 7.4), and bovine serum albumin (BSA, $1 \% \mathrm{w} / \mathrm{v}$ in TE). Sulfo-LC-SPDP (sulfosuccinimidyl 6-[3'-(2-pyridyldithio)propionamido] hexanoate), the linker for surface immobilization, was acquired from Pierce. $250 \mathrm{~nm}$ Micromod nanomag ${ }^{\circ}$-D magnetic particles have a magnetite core and a dextran shell, are available in a $4.9 \times 10^{11}$ particles $/ \mathrm{mL}$ concentration, and with a surface modified with streptavidin, which is suitable for streptavidinbiotin functionalization with biotinylated antibodies.

\subsection{Bacterial strain and polyclonal antibodies}

Biosafety Level 3 facilities are required in Mtb bacteria manipulation. Therefore, an attenuated strain used as a vaccine against TB was applied as a safe surrogate for Mtb: Mycobacterium bovis Bacillus Calmette-Guérin (BCG) Pasteur from the Trudeau Institute Culture Collection. BCG samples were grown to mid-log phase in ProskauerBeck medium with $0.05 \%$ Tween 80 and frozen at $-70{ }^{\circ} \mathrm{C}$ (Cruz et al., 2010). Then, a loopful of BCG resuspended in PB buffer was treated as described by Bettencourt and co-workers to obtain $1 \times 10^{7}$ bacteria per $\mathrm{mL}$, at an optical density of $600 \mathrm{~nm}$ (Bettencourt et al., 2010). Polyclonal antibodies (Abs), from Thermo Fisher Scientific, biotin conjugated anti-M. tuberculosis (anti-Mtb bio, PA1-73136, 4-5 mg/ $\mathrm{mL}$ ), anti-M. tuberculosis (anti-Mtb, PA1-7260, 4-5 mg/mL) and antiSalmonella (anti-Salm, PA1-20811, $1 \mathrm{mg} / \mathrm{mL}$ ), were washed from their preservative solution by filtering with Millipore centrifugal filters (100 kDa cut-off, $10.000 \mathrm{rpm}, 15 \mathrm{~min}$ ) and exchanged to PB buffer to the desired concentration. The Abs concentration was optimized in previous studies (Barroso et al., 2015).

\subsection{Bioconjugation of MNP with anti-Mtb bio and BCG}

Streptavidin capped MNP, with a $10^{6} \mathrm{NP} / \mu \mathrm{L}$ concentration, were magnetically washed 3 times with $\mathrm{PB}$, using a magnetic concentrator (Dynal-biotech), and then allowed to react with $25 \mu \mathrm{g} / \mathrm{mL}$ of anti-Mtb bio, in a total volume of $200 \mu \mathrm{L}$. This solution was incubated $1 \mathrm{~h}$, at $200 \mathrm{rpm}$ and room temperature (RT). After Abs immobilization on the
MNP, free reactive sites on MNP surface were blocked with BSA in TE for $30 \mathrm{~min}$, at $200 \mathrm{rpm}$ and RT, to avoid non-specific adsorption of other proteins or false positive results. Then, functionalized MNP were magnetically washed 3 times with $\mathrm{PB}$. The magnetic capture of BCG was performed by incubating $200 \mu \mathrm{L}$ of different concentrations of BCG cells $\left(0 / 10^{4} / 10^{5} / 10^{6} / 10^{7}\right.$ cells $\left./ \mathrm{mL}\right)$ with MNP@Abs, for $1 \mathrm{~h}$, at $200 \mathrm{rpm}$ and RT, resulting in MNP@Abs@BCG detection probes. Then, each concentration level was magnetically washed 3 times with $\mathrm{PB}$ and re-suspended in PB until further use.

Optimization studies, regarding the efficiency of the magnetic capture and further recognition of BCG bacteria by capture Abs on the MR-biochip surface, were conducted in a previous work (Barroso et al., 2015).

\subsection{Bioconjugated MNP immobilization on the MR-biochip}

The MR-biochip was developed by INESC MN and the probe immobilization surface consists of patterned $\mathrm{Cr} / \mathrm{Au}$ pads, with MR sensors beneath. Basically, the MR-biochip includes two separate gold sensing areas, each one with 3 groups of $5 \mathrm{U}$-shaped $2.5 \times 80 \mu^{2}$ spinvalve sensors, in a total of 30 active testing sensors (Martins et al., 2009). After rigorous cleaning of the surface to remove the protective resist layer and residues, a droplet of $20 \mu \mathrm{L}$ of sulfo-LC-SPDP, a heterobifunctional cross-linker for immobilization with amino groups, was placed over the surface for $1 \mathrm{~h}$, at RT, in a humidified atmosphere (HA) (Fernandes et al., 2014). The surface was then rinsed with PB and ultrapure water, and gently dried with a nitrogen gun. Then, droplets of $1 \mu \mathrm{L}$ of both negative control (anti-Salm) and positive sample (antiMtb), both with a $50 \mu \mathrm{g} / \mathrm{mL}$ concentration, were manually spotted on the surface, respectively on the left and right sides, and incubated for $2 \mathrm{~h}$, at RT, in HA. To remove unbound Abs and block nonspecific adsorption, the gold surface was rinsed with $\mathrm{PB}$, and $20 \mu \mathrm{L}$ of BSA in TE were allowed to react $1 \mathrm{~h}$, at RT, in HA. Finally, the surface was rinsed with $\mathrm{PB}$ to remove the excess of blocking solution.

\subsection{MR-biochip measurement}

The MR-biochip is a plugged-in electronic platform (Germano et al., 2009). The device has a microfluidic channel controlled by a syringe pump (New Era NE-300) that controls and loads the sample into the MR-biochip. Measurements were performed with $1 \mathrm{~mA}$ biasing current for the sensors, and an external $3 \mathrm{mT}$ magnetic bias field and $1.35 \mathrm{mT}$ rms AC field for magnetizing the MNP. The baseline signal acquisition was provided by $15 \mathrm{~min}$ of PB buffer flow. Then, $5 \mu \mathrm{L}$ of MNP@Abs@BCG in PB were introduced and settle down on the surface of the sensor for $30 \mathrm{~min}$. Finally, two washing steps with PB buffer allowed the removal of unbound MNP, first at a flow rate of $5 \mu \mathrm{L} / \mathrm{min}$ for $5 \mathrm{~min}$, and then $50 \mu \mathrm{L} / \mathrm{min}$ for $5 \mathrm{~min}$. All throughout this process, the signal acquisition was sequentially measured for all sensors inside the MR-biochip. The $\Delta \mathrm{V}_{\text {binding }}$ in each sensor was obtained by the voltage differential between the baseline signal $\left(\mathrm{V}_{\text {sensor }}\right)$ and the binding signal in the $50 \mu \mathrm{L} / \mathrm{min}$ washing step $\left(\mathrm{V}_{\text {particles }}\right): \Delta \mathrm{V}_{\text {binding }}=$ $\mathrm{V}_{\text {sensor }}-\mathrm{V}_{\text {particles }}$. This differential is proportional to the number of magnetically labelled mycobacteria, successfully bound to the surface. The $\Delta \mathrm{V}_{\text {binding }}$ is then normalized to each sensor baseline signal $\left(\mathrm{V}_{\text {sensor }}\right)$ at the measurement conditions $\left(\Delta \mathrm{V}_{\text {binding }} / \mathrm{V}_{\text {sensor }}\right)$, to compare different sensors in the same assay and among different MR-biochips (Martins et al., 2009).

\subsection{Data processing}

\subsubsection{Biochip specificity}

Biochip specificity (binding affinity) was tested by comparing the signal intensities between positive and negative sensors, at different BCG bacteria concentrations (five levels: $0 / 10^{4} / 10^{5} / 10^{6} / 10^{7}$ cells $/ \mathrm{mL}$ ). Student's $t$-test was performed to determine signal differences at each 
level. Fisher's test (F-test) was performed to evaluate tendency between variance of positive and negative signals (Knuter et al., 2005).

\subsubsection{Multiplicative signal correction}

Multiplicative signal correction (MSC) was used to correct baseline and proportional systematic effects (Martens and Stark, 1991; Martens et al., 2003; Gallagher et al., 2005). Both effects can be corrected by:

$\mathrm{V}_{\text {cor }}=V \beta$

Where $\mathrm{V}_{\text {cor }}$ is the corrected voltage, $\mathrm{V}$ is the recorded voltage and $\beta$ are the correction coefficients calculated by minimizing the distance to a reference voltage profile $\left(\mathrm{d}_{\mathrm{J}}\right)$.

$d_{J}=\beta_{1} V_{j}+\beta_{2}-V_{\text {ref }}$

Where $d_{j}$ is the distance, $V_{j}$ is the voltage profile of sample $j$ and $V_{\text {ref }}$ is the mean reference voltage profile. Once the error is minimized, $V$ is corrected into $\mathrm{V}_{\text {cor }}$, without the effects of different baselines and proportional systematic multiplicative effects. $\mathrm{V}_{\text {cor }}$ is afterwards used in the calibration curves. For any new biochip TB measurement, the correction is applied before quantification, using the recorded mean reference voltage profile.

\subsubsection{Biochip linear calibration}

Biochip calibration is the process of finding a relevant statistical relationship between MR voltage and cell concentration. Magnetoresistance increases non-linearly with BCG cell concentration (CTB). A log-linear function is observed: $\log _{10}$ (CTB) $=\mathrm{f}\left(\Delta \mathrm{V}_{\text {binding }} /\right.$ $\mathrm{V}_{\text {sensor }}$ ).

Linear regression was performed using the least-squares optimization method. Regression coefficients were analyzed for statistical significance using the t-student statistics, and linearity was assessed by analysis of variance (ANOVA) for determining the p-value of the linear relationship (Knuter et al., 2005). The LOD was considered 10 fold of the regression standard error.

The standard deviation (SD) and coefficient of variation (CV) of the voltage drop $\left(\Delta \mathrm{V}_{\text {binding }} / \mathrm{V}_{\text {sensor }}\right)$, for each MR-biosensor at different BCG cell concentrations were calculated, determining the reproducibility of the biochips at each level. Bias was calculated as the difference from the linear model prediction and the actual concentration value.

\subsubsection{Principal component regression}

Principal component regression (PCR) is a multivariate calibration that uses the biochip voltage profile to establish a linear relationship between cell concentration and projection of scores into the principal components. PCR relates the orthogonally decomposed variance in the voltage signal profile with the cell concentration. The inclusion of more than one relevant principal component in the PCR regression denotes non-random variation in the voltage signal, which contains information about cell concentration. Once more systematic information is available to relate cell concentration, less bias and variance is expected in the MR-biochip calibration (Hastie et al., 2009).

\subsubsection{Partial least-squares regression}

Partial least-squares regression (PLS) maximizes the co-variance between biochip voltage (V) and cell concentration (C); and their corresponding latent variables (LV). In PLS, the co-variance matrix is orthogonally decomposed so that it maximizes each LV correlation. PLS coefficients are obtained using only the latent dimensions that are able to represent information of projection of $\mathrm{C}$ in $\mathrm{V}$. The optimized coefficients are obtained at the number of LV that minimizes the crossvalidation prediction error sum of squares (Lindgren et al., 1993; Wold et al., 2001; Trygg and Wold, 2002).

\subsubsection{Validation}

All models (linear regression, PCR and PLS) were obtained using cross-validation with the "leave-one-out" methodology. The standard error (SE) was obtained by summing the prediction error of each sample that was left out of the training batch (Knuter et al., 2005). The number of LV, in both PCR and PLS, was determined by minimizing the mean prediction square error, obtaining a stable multivariate model that correctly balances bias vs. variance (Sutter et al., 1992; Wentzell and Montoto, 2003; Hastie et al., 2009). Model coefficients variance and $t$-test were obtained by bootstrapping and re-sampling (Manly, 1998; Knuter et al., 2005). The coefficient of determination (Radj) and Pearson correlation $\left(R^{2}\right)$ were used as metrics of tendency. The p-value of all models was assessed by the F-test between measured and predicted values (Knuter et al., 2005). All statistical models were performed using R (R-project, 2017).

\section{Results and discussion}

\subsection{Biochip specificity}

Despite the fact that anti-Salm Abs showed some unspecific binding events, the signal obtained in the positive sensors (anti-Mtb) was always higher and statistically different from the signal coming from the negative control sensors (anti-Salm).

Fig. 2 presents the MR signal intensities after washing, for anti-Mtb positive sample and anti-Salm negative control, at different concentrations of BCG cells $\left(10^{4}\right.$ to $10^{7}$ cells $\left./ \mathrm{mL}\right)$, as well as, the zero level signal ( 0 cells $/ \mathrm{mL}$ ). The MR signals of anti-Mtb positive samples are higher than the zero level signals, for all cell concentrations. The anti-Salm negative sensors always present lower MR signals $\left(\Delta \mathrm{V}_{\text {binding }}\right)$, for any cell concentration, than the positive anti-Mtb sensors. Even at the lowest concentration $\left(10^{4}\right.$ cells $\left./ \mathrm{mL}\right)$, the voltage differential $\left(\Delta \mathrm{V}_{\text {binding }} /\right.$ $\mathrm{V}_{\text {sensor }}$ ) of negative controls is significantly lower than the positive samples $\left(\mathrm{p}<10^{-3}\right)$. All positive samples have significantly higher values than both zero level signal and negative control $\left(\mathrm{p}<10^{-3}\right)$. In both negative controls and positive samples, the signal intensity increases with concentration, as well as, the difference between control and sample signals.

The MR signal in positive samples is always higher than in negative controls $\left(\mathrm{p}<10^{-3}\right)$. Nevertheless, total specificity is apparently not achieved. The negative signal increases with BCG concentration, which is a sign of non-specific binding between anti-Salm control Abs and BCG bacteria. This non-specific binding is linearly correlated to BCG concentration, and therefore, it is a systematic limitation of the MRbiochip, not affecting performance, since the proportion is maintained across the different replicates. There is a direct linear relationship between the signal increase of negative control and positive samples ( $\mathrm{p}$ $<0.05)$. Such is a good indication that non-specific binding is linearly

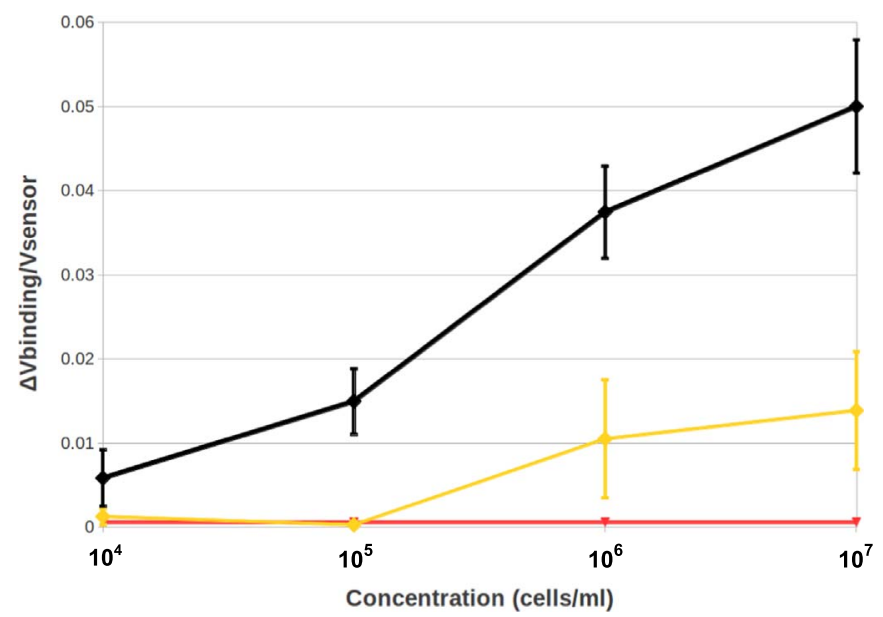

Fig. 2. Biochip specificity in raw data: differences between signal intensities of anti-Mtb positive samples and anti-Salm negative controls (black - positive sample; yellow negative control; red - maximum background from 0 cells $/ \mathrm{mL}$ ). 
a

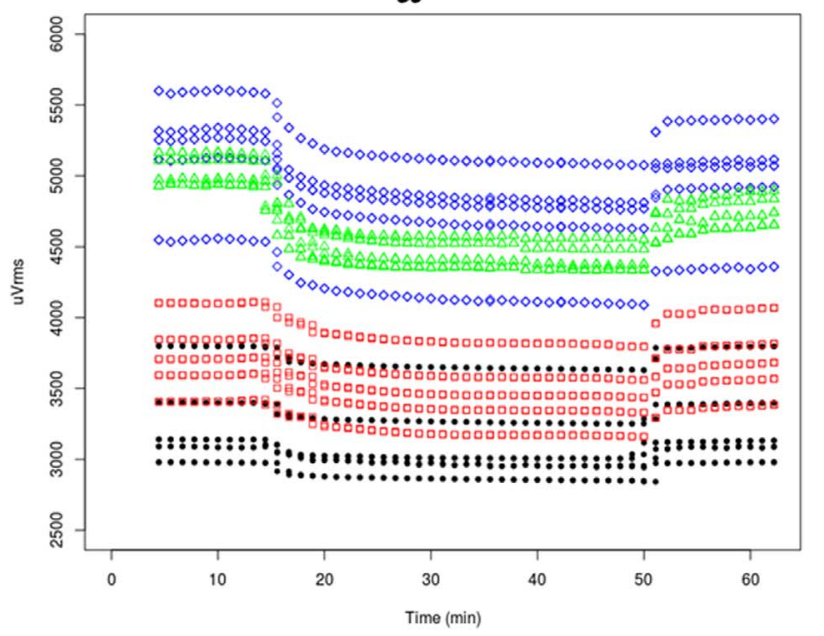

Cell concentration (cells $/ \mathrm{mL}$ ):

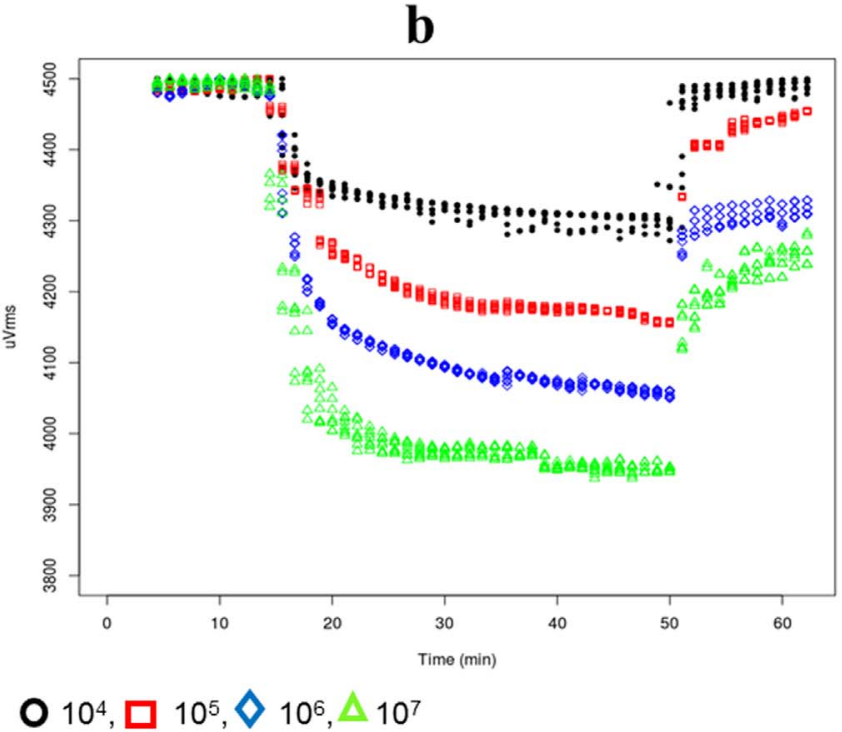

Fig. 3. Voltage mean signal correction: a) raw biochip voltage curves; b) corrected biochip voltage curves.

related to BCG concentration, and should not affect the quality of TB quantification. This effect has been already observed in previous studies (Martins et al., 2009; Barroso et al., 2015).

\subsection{Multiplicative signal correction}

Fig. 3 shows how MSC improves the original MR-biochip signal. Fig. 3a shows raw data and Fig. 3b presents data after MSC.

The raw data indicates that the initial baseline is not the same for the different sensors and replicates. For each cell concentration, the baseline variance is significant, with an average CV of $6.1 \%$ (Table 1). The same is observed after washing, where the signal has an average $\mathrm{CV}$ of $6.1 \%$. This variation masks the direct comparison of the voltage profile at different cell concentrations (Fig. 3a).

After MSC, the MR signal at different concentration levels is clearly visible (Fig. 3b). All baselines are corrected to a similar value with an average of $4489.74 \mu \mathrm{V}$, and extremely low variance (average CV 0.11\%). Moreover, the voltage in washing decreases with BCG cell concentration levels, which was not directly observable in the original signal, because systematic effects were uncorrected. Also, the voltage in washing reaches low variance (average CV $0.4 \%$, see Table 1 ). MSC on MR-biochip proves to be extremely effective. The voltage signal, both in baseline and washing, is corrected and maintains proportionality to BCG cell concentration. The corrected voltage profile is now distinguishable for different cell concentrations, which is an indication that MSC preserves the information about differential effects and binding reactions at the MR-biochip surface.

Results show that the voltage potential $\left(\Delta \mathrm{V}_{\text {binding }}\right)$ increases linearly with the decimal logarithm of cell concentration: $\log _{10}($ cell $/ \mathrm{mL})=$ $\mathrm{f}(\Delta \mathrm{V})$. The direct calibration using the normalized raw data has a standardize error (SE) of $0.5 \log _{10}($ cells $/ \mathrm{mL})$ and $\mathrm{r}^{2}=0.8145(\mathrm{p}<$ $10^{-9}$ ). Quantification using the direct voltage had significant error, because systematic errors were left uncorrected. An SE of 0.5 is only satisfactory for providing qualitative TB data classification, for example, the CV of the baseline or washing was around $10 \%$, which inevitably led to high dispersion in the direct calibration curve (Table 1). Once the original signal has been corrected by MSC, SE drops to $0.03309\left(\mathrm{r}^{2}=0.95562\right)$. Such is a very significant improvement, as the CV at $10^{4}$ cells $/ \mathrm{mL}$ is now only $8.27 \%$, providing data with quantification capacity within $10^{4}$ to $10^{7}$ cells $/ \mathrm{mL}$.

The effect of MSC is also directly visible in Fig. 4a. This strategy significantly reduces the variance dispersion of the voltage drop in washing, when compared to non-MSC correction, as presented in the raw data of Fig. 2.

\subsection{Principal component regression and Partial least-squares regression}

MSC has the same effect on the PCR model. Raw data only allows to obtain a PCR model with high variance $\left(\mathrm{SE}=0.5325 ; \mathrm{r}^{2}=0.8229, \mathrm{LV}=\right.$ 1), whereas, MSC allows a PCR model with well-balanced bias-variance ( $\mathrm{SE}=0.1245, \mathrm{r} 2=0.9924, \mathrm{LV}=2)$. Such is a significant improvement against the zeroing or normalization methods. With an extremely low MAPE at the lower concentration (3.11\%), the MR-biochip can now reach analytical quality in TB quantification from $10^{4}$ to $10^{7}$ cell $/ \mathrm{mL}$ (ISO, 2006; Westgard, 2015). In Table 2 is possible to observe that the SE of PCR calibration is 0.1245 , a very significant decrease when compared with the traditional normalization or non-MSC corrected signal (SE 0.5).

PLS achieves better results, obtaining analytical quality calibration curve with $r^{2}=0.9943$ and MAPE $<1 \%$ (Table 2). PLS outperforms PCR, because it maximizes co-variance between cell concentration and voltage signal, extracting the regression coefficients that linearity relate the voltage curve pattern to cell concentration.

Table 1

Voltage variation of the magnetoresistive biochip.

\begin{tabular}{|c|c|c|c|c|}
\hline \multirow[t]{2}{*}{ Concentration (cells/mL) } & \multicolumn{2}{|c|}{ Original Signal $(\mu \mathrm{V})$} & \multicolumn{2}{|c|}{ Corrected Signal $(\boldsymbol{\mu V})$} \\
\hline & Baseline & Washing & Baseline & Washing \\
\hline $10^{4}$ & $3342.99 \pm 305.45$ & $3338.95 \pm 303.86$ & $4497.20 \pm 5.34$ & $4491.74 \pm 7.13$ \\
\hline $10^{5}$ & $3783.22 \pm 248.29$ & $3750.38 \pm 243.89$ & $4486.26 \pm 3.79$ & $4447.43 \pm 5.62$ \\
\hline $10^{6}$ & $5103.12 \pm 347.89$ & $4915.04 \pm 342.16$ & $4481.51 \pm 6.12$ & $4311.45 \pm 11.07$ \\
\hline $10^{7}$ & $5072.98 \pm 102.27$ & $4783.95 \pm 89.61$ & $4493.97 \pm 5.32$ & $4228.09 \pm 45.11$ \\
\hline
\end{tabular}



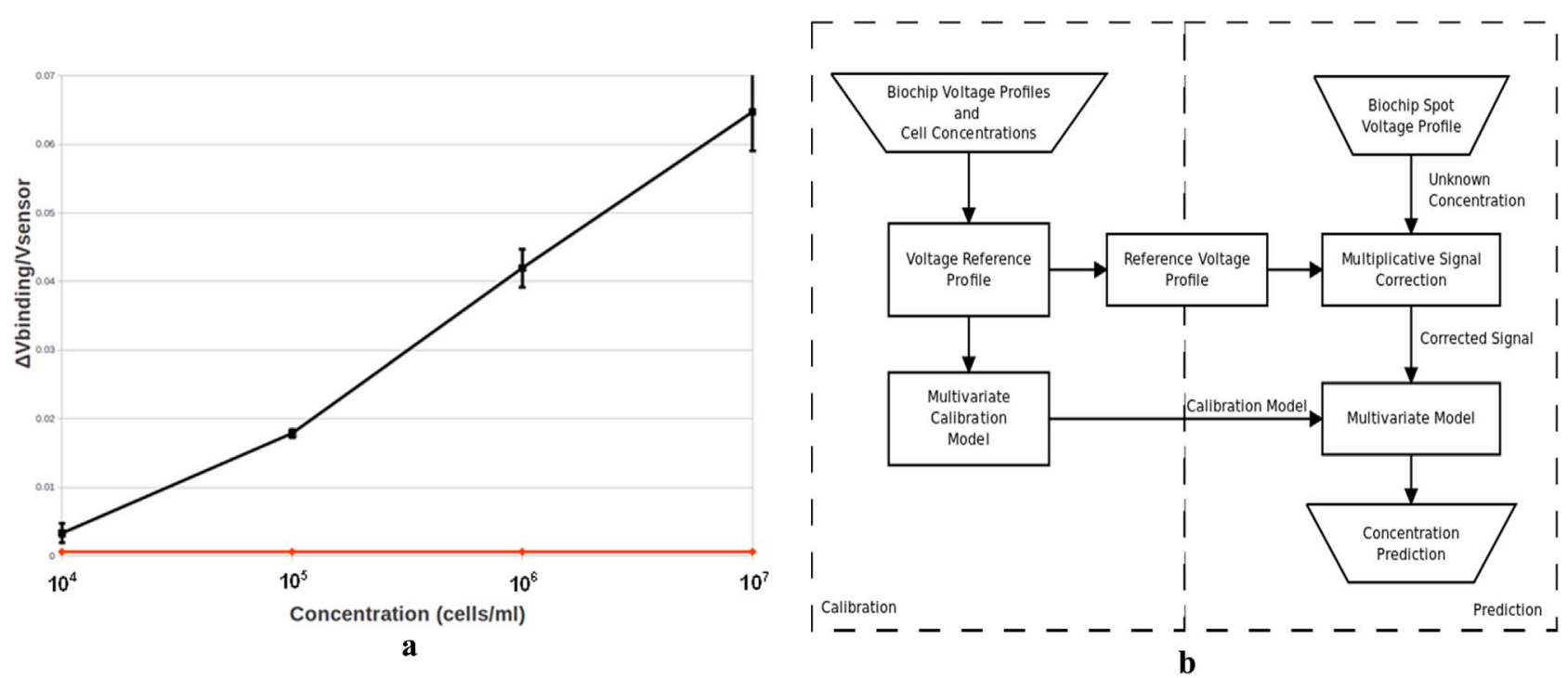

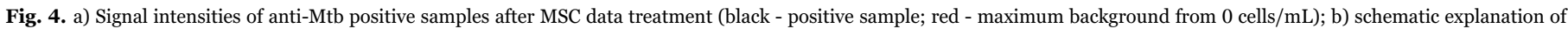
the biochip multivariate chemometrics method.

These results suggest that the voltage curve has significant information that can be used to quantify BCG cells. Even after MSC correction, using a linear model of the voltage drop in washing, the SE is 0.3309 , which is ten times greater than the PLS approach. These results allow concluding that the main variance of voltage profile contains systematic information about BCG concentration and should be explored in MRbiochip technology to obtain better quantitative models.

Furthermore, the calculation of $\Delta \mathrm{V}_{\text {binding }} / \mathrm{V}_{\text {sensor }}$ introduces variance. As the voltage profile in washing is also dependent on diffusion and fluid flow, the voltage difference between baseline and washing is not totally reproducible, even after MSC correction.

Because the dynamics of diffusion and reaction are recorded in the voltage profile, the MR-biochip fabrication and sample preparation should be kept reproducible, so that chemometrics methodology is effective in MR-biochips, as we suggest in Fig. 4b:

- Calibration step: i) perform measurements with MR-biochips from different production lots, and register the voltage profiles for different concentrations; ii) perform MSC to all voltage profiles and register the reference voltage profile; and iii) build the calibration model;

- Prediction step for unknown samples: i) record the voltage profile for an unknown sample; ii) correct the signal with MSC using the reference voltage profile; and iii) predict the BCG concentration using the calibration model.

\subsection{Calibration and detection limit}

The LOD allows us to explore the limits of the MR-biochip technology for TB diagnosis. The SE of MSC-PCR and MSC-PLS calibrations allow LOD of $13.3 \mathrm{cell} / \mathrm{mL}$ and 10.8 cell $/ \mathrm{mL}$, respectively. Such low LOD allows the extrapolation that the MR-biochip is able to measure BCG bellow $10^{4}$ cells/mL, without compromising the analytical quality of the quantification. This was the initial objective of the study, because $\mathrm{ZN}$ can go as low as $10^{4}$ cells $/ \mathrm{mL}$.

Although calibration results show very low SE, the MR-biochip needs to improve: i) functionalization protocol; and ii) non-specific binding. The current MNP@Abs@BCG functionalization protocol involves too many manual steps of magnetic separation and washing. In each step there is a small loss of MNP, which at the end of the bioconjugation, contributes to a slightly different content of MNP in each level of BCG concentration. This variation introduces systematic errors in the measurement, reducing the reproducibility of the method.

Furthermore, different MR-biochip fabrication batches will always present small differences. Most of the biochip signal variability arises from: i) MNP interference; ii) Abs density and distribution on the biochip surface; iii) electronics fabrication; and iv) diffusional effects on the biochip surface. Also, systematic effects from the MR-biochip fabrication (e.g. biochip area and MR sensitivity), result in the observed voltage curves in Fig. 3a, in cell concentration levels from $10^{4}$ to $10^{7}$ cells/mL. Ideally, the baseline signal should be identical for every MRbiochip measurement, and the voltage drop should be similar for the same cell concentration.

Procedures to decrease non-specific binding, as well as, decreasing the deposition of cells at the sensor surface area with no antibodyantigen immobilization, by increasing the washing steps and improving washing buffers efficacy, would be recommended for better efficiency and reproducibility. Moreover, specific interference tests, cross-reactivity tests, as well as, automatizing the magnetic separation and washing steps during functionalization should greatly reduce systema-

Table 2

Biochip calibration results.

\begin{tabular}{|c|c|c|c|c|c|}
\hline Model & Data & Type & $\mathbf{S E}^{\mathrm{a}}$ & $\mathbf{R}^{2 \mathrm{~b}}$ & p-value ${ }^{c}$ \\
\hline Linear I & Raw normalized voltage & Linear least squares regression & 0.5046 & 0.8145 & $<1 \times 10^{-9}$ \\
\hline Linear II & Corrected voltage & Linear least squares regression & 0.3309 & 0.9562 & $<1 \times 10^{-9}$ \\
\hline PCR I & Raw normalized voltage & Principal component regression & 0.5325 & 0.8229 & $<1 \times 10^{-9}$ \\
\hline PCR II & Corrected voltage & Principal component regression & 0.1245 & 0.9924 & $<1 \times 10^{-16}$ \\
\hline PLS I & Corrected voltage & Partial least squares & 0.0322 & 0.9993 & $<1 \times 10^{-16}$ \\
\hline
\end{tabular}

a Standard Error: blind tests error.

b Regression correlation coefficient.

${ }^{\mathrm{c}}$ Fisher's test p-value. 
tic errors. Also, random sampling tests could be done, using process analytical techniques, such as multi-variate control charts, which can be implemented for controlling the quality of different MR-biochips batches (Mitsutake et al., 2015).

\subsection{Benchmark with the state-of-the-art}

The benchmark LOD on biosensors for TB diagnosis is currently $8 \times$ $10^{2}$ cells $/ \mathrm{mL}$, obtained with an electrochemical biosensor based in a microtip immunoassay for H37Ra cells (Yeo et al., 2010). Electronic nose technology achieved a LOD of $10^{4}$ in RIVM myc 4514 cells (Fend et al., 2006). Mechanical biosensors in attenuated Mtb cells achieved LOD of $2 \times 10^{3}$ (He et al., 2003) and $10^{5}$ (He and Zhang, 2002), respectively, with acoustic wave and quartz crystal microbalance. In optical biochips, LOD was $10^{3}$ and $10^{4}$ cells $/ \mathrm{mL}$ with Mtb sputum samples, respectively, when using isothermal loop mediated amplification (Creecy et al., 2015) and capture ELISA of lipoarabinomannan antigen (Arias-Bouda et al., 2000). Similar LOD results, $10^{3}$ and $10^{4}$, were achieved by magnetic methods, respectively, by using a magnetic barcode in sputum (Liong et al., 2013) and a magnetoelastic biosensor in Mtb H37Ra strain cells (Pang et al., 2008).

The SE obtained by PCR and PLS models (Table 2) allows concluding that the MR-biochip technology is a potential candidate to replace $\mathrm{ZN}$ in POC context. If one considers that the LOD is $10 \%$ the SE, we deduce that the estimated LOD can achieve 10.8 cells/mL in the PLS model and 13.3 cells $/ \mathrm{mL}$ in the PCR model, which is below $10^{4}$ cells $/ \mathrm{mL}$.

\section{Conclusions}

This research presents a proof-of-concept POC MR-biosensor platform for TB diagnosis. Results show that, although the binding of BCG is not $100 \%$ specific, the difference of the MR voltages between positive sample and negative control or zero level background is always statistically significant.

Results also demonstrate that systematic artifacts, due to hardware limitations in MR-biochips, are effectively corrected using MSC techniques. Furthermore, when MSC is coupled with chemometrics modelling (PCR and PLS), significant reproducibility and LOD gains are obtained. Therefore, we propose the implementation of an algorithm to be used in the MR-biosensor technology.

In conclusion, the low LOD of the MR-biosensor presents high potential for TB diagnosis at the point-of-care. However, MR-biochip technology still relies on pre-processing of samples before measurements. Future research on the extraction and isolation procedures of clinical samples will allow the use of the presented technology in real world applications.

\section{Acknowledgments}

Teresa Barroso thanks FCT for her PhD Grant SFRH/BD/33904/ 2009. Elisabete Fernandes acknowledges the Project N2020 -PEAdvancing Cancer (NORTE-01-0145-FEDER-000029).

\section{References}

Arias-Bouda, L.M.P., Nguyen, L.N., Ho, L.M., Kuijper, S., Jansen, H.M., Kolk, A.H., 2000. J. Clin. Microb. 38 (6), 2278-2283.

Barroso, T.R.G., Martins, V.C., Cardoso, F., Cardoso, S., Pedrosa, J., Correia-Neves, M., Rivas, J., Freitas, P.P., 2015. JNanoR 34, 49-60.

Bettencourt, P., Pires, D., Carmo, N., Anes, E., 2010. Application of confocal microscopy for quantification of intracellular mycobacteria macrophages. In: Méndez-Vilas, A., Díaz, J. (Eds.), Microscopy: Science, Technology, Applications and Education 1. Formatex Research Center, Badajoz, 614-621.

Creecy, A., Russ, P.K., Solinas, F., Wright, D.W., Haselton, F.R., 2015. PLOS One. 10 (7), e0130260.

Cruz, A., Fraga, A.G., Fountain, J.J., Rangel-Moreno, J., Torrado, E., Saraiva, M., Pereira,
D.R., Randall, T.D., Pedrosa, J., Cooper, A.M., Castro, A.G., 2010. J. Exp. Med. 207 (8), 1609-1616.

Fend, R., Kolk, A.H.J., Bessant, C., Buijtels, P., Klatser, P.R., Woodman, A.C., 2006. J. Clin. Microb. 44 (6), 2039-2045.

Fernandes, E., Martins, V.C., Nóbrega, C., Carvalho, C.M., Cardoso, F.A., Cardoso, S., Dias, J., Deng, D., Kluskens, L.D., Freitas, P.P., Azeredo, J., 2014. Biosens. Bioelectron. 52, 239-246.

Freitas, P.P., Cardoso, F.A., Martins, V.C., Martins, S.A.M., Loureiro, J., Amaral, J., Chaves, R.C., Cardoso, S., Fonseca, L.P., Sebastião, A.M., Pannetier-Lecoeur, M., Fermon, C., 2012. Lab Chip 12 (3), 546-557.

Freitas, P.P., Martins, V.C., Cardoso, F.A., Fernandes, E., Sobrino, T., Castillo, J., Chicharo, A., Abal, M., Lopez-Lopez, R., Dias, T.S., Cardoso, S., 2016. Spintronic biochips: from the laboratory to pre-clinical applications. In: Fermon, C., Van DeWorde, M. (Eds.), Nanomagnetism: Applications and Perspectives. John Wiley and Sons, New York, 165-200.

Gallagher, N.B., Blake, T.A., Gassman, P.L., 2005. J. Chemom. 19 (5-7), 271-281.

Germano, J., Martins, V.C., Cardoso, F.A., Almeida, T.M., Sousa, L., Freitas, P.P., Piedade, M.S., 2009. Sensors 9 (6), 4119-4137.

Giouroudi, I., Keplinger, F., 2013. Int. J. Mol. Sci. 14, 18535-18556.

Hastie, T., Robert, T., Jerome, F., 2009. The Elements of Statistical Learning - Data Mining, Inference, and Prediction. Springer-Verlag, New York.

He, F.J., Zhang, L.D., 2002. Anal. Sci. 18 (4), 397-401.

He, F.J., Zhao, J.W., Zhang, L.D., Su, X.N., 2003. Talanta 59, 935-941.

ISO 22870, 2006. Point of care testing - requirements for quality and competence. International Organization for Standardization, Geneva.

Knuter, M.H., Nachtsheim, C.J., Neter, J., Li, W., 2005. Applied Linear Statistical Models fifth ed.. McGraw-Hill, Boston.

Lange, C., Mori, T., 2010. Respirology 15 (2), 220-240.

Lindgren, F., Geladi, P., Wold, S., 1993. J. Chemom. 7, 45-59.

Liong, M., Hoang, A.N., Chung, J., Gural, N., Ford, C.B., Min, C., Shah, R.R., Ahmad, R., Fernandez-Suarez, M., Fortune, S.M., Toner, M., Lee, H., Weissleder, R., 2013. Nat. Commun. 4, 1752.

Manly, B.F.J., 1998. Randomization, Bootstrap and Monte Carlo Methods. In: Biologysecond ed.. Chapman and Hall, U.S.A.

Martens, H., Stark, E., 1991. J. Pharm. Biomed. Anal. 9 (8), 625-635.

Martens, H., Nielsen, J.P., Engelsen, S.B., 2003. Anal. Chem. 75 (3), 394-404.

Martins, V., Fonseca, L.P., Ferreira, H.A., Graham, D.L., Freitas, P.P., Cabral J.S., 2005 Technical Proceedings of the NSTI Nanotechnology Conference and Trade Show 1, 493-496.

Martins, V.C., Cardoso, F.A., Germano, J., Cardoso, S., Sousa, L., Piedade, M., Freitas, P.P., Fonseca, L.P., 2009. Biosens. Bioelectron. 24 (8), 2690-2695.

Martins, V.C., Germano, J., Cardoso, F.A., Loureiro, J., Cardoso, S., Sousa, L., Piedade, M., Fonseca, L.P., Freitas, P.P., 2010. J. Magn. Mag. Mater. 322 (9-12), 1655-1663.

Martins, S.A., Martins, V.C., Cardoso, F.A., Freitas, P.P., Fonseca, L.P., 2012. Waterborne pathogen detection using a magnetoresistive immuno-chip. In: Tiquia-Arashiro, S.M. (Ed.), Molecular Biological Technologies for Ocean Sensing. Springer Protocols Handbooks, New York, 263-288.

Matu, W., Juma, E., 2013. J. Life Sci. 7 (6), 607-612.

Moreno-Pérez, D., Martín, A.A., Gómez, N.A., Baquero-Artigao, F., Montaner, A.E., Durán, D.G., Montero, R.G., Peña, M.J.M., Rodrigo-Gonzalo-de-Liria, C., Serrano, M.J.R., 2010. Anal. Ped. 73, 143.1-143.14.

Mitsutake, H., Guimarães, E., Freitas, H.C., Gontijo, L.C., Santos, D.Q., Neto, B.W., 2015 J. Chemom. 29, 411-419.

Morton, T.A., Myszka, D.G., 1998. Methods Enzymol. 295, 268-294.

Myszka, D.G., 1999. J. Mol. Recognit. 12 (5), 279-284.

Pang, P., Cai, Q., Yao, S., Grimes, C.A., 2008. Talanta 76 (2), 360-364.

Parsons, L.M., Somoskövi, A., Gutierrez, C., Lee, E., Paramasivan, C.N., Abimiku, A., Spector, S., Roscigno, G., Nkengasong, J., 2011. Clin. Microbiol Rev. 24 (2), 314-350.

Perkins, M.D., Cunningham, J., 2007. J. Infect. Dis. 196, S15-S27.

R-project, 2017. The R project for statistical computing. URL: 〈http://www.r-project. $\mathrm{org} /\rangle$ (Accessed 30 May 2017).

Sutter, M., Kalivas, J.H., Patrick, M.L., 1992. J. Chemom. 6 (4), 217-225.

Trygg, J., Wold, S., 2002. J. Chemom. 16 (3), 119-128.

Veigas, B., Doria, G., Baptista, P.V., 2012. Nanodiagnostics for Tuberculosis. In: Cardona, P. (Ed.), Understanding Tuberculosis - Global Experiences and Innovative Approaches to the Diagnosis. InTech, Rijeka, Croatia, 257-276.

Wentzell, P.D., Montoto, L.V., 2003. Chemom. Intel. Lab. Syst. 65 (2), 257-279.

Westgard J., 2015. CLIA Requirements for Analytical Quality. URL: 〈https://www. westgard.com/clia.htm\#he (Accessed 30 May 2017).

Wold, S., Sjöströma, M., Erikssonb, L., 2001. Chemom. Intel. Lab. Syst. 58 (2), 109-130.

World Health Organization, 2012. In: WHO (Ed.), Global Tuberculosis Report 2012. WHO, Geneva.

World Health Organization, 2015. In: WHO (Ed.), Global Tuberculosis Report 2015. WHO, Geneva.

World Health Organization, 2016. In: WHO (Ed.), Global Tuberculosis Report 2016. WHO, Geneva.

Yeo, W.H., Liu, S., Chung, J.H., Liu, Y., Lee, K.H., 2009. Anal. Bioanal. Chem. 393 (6-7), 1593-1600.

Yeo, W.H., Chou, F.L., Fotouhi, G., Oh, K., Stevens, B.T., Tseng, H.Y., Gao, D., Shen, A.Q., Chung, J.H., Lee, K.H., 2010. Lab Chip 10 (22), 3178-3181.

Zhang, H., Williams, P.S., Zborowski, M., Chalmers, J.J., 2006. Biotechnol. Bioeng. 95 (5), 812-829. 